\title{
Multiple Cranial Neuropathies as the Presenting Sign in a Patient with Metastatic BRAF-Mutated Lung Adenocarcinoma with Leptomeningeal Involvement
}

\author{
Bailey Gleason Fitzgerald ${ }^{a} \quad$ Michael Grant ${ }^{b} \quad$ Gbambele Kone $^{c}$ \\ Huned Patwa $^{\mathrm{d}}$ Michal Rose ${ }^{\mathrm{e}}$ \\ aYale University School of Medicine, Yale-New Haven Hospital and Veterans Affairs \\ Connecticut Healthcare System, New Haven, CT, USA; bYale University School of Medicine \\ and Smilow Cancer Center, Yale-New Haven Hospital and Veterans Affairs Connecticut \\ Healthcare System, New Haven, CT, USA; ' Yale University School of Medicine Department \\ of Neurology, New Haven, CT, USA; dYale University School of Medicine and Veterans \\ Affairs Connecticut Healthcare System Department of Neurology, New Haven, CT, USA; \\ eYale University School of Medicine and Smilowa Cncer Center, Veterans Affairs Connecticut \\ Healthcare System, West Haven, CT, USA
}

\section{Keywords}

Non-small cell lung cancer - BRAF-mutated lung adenocarcinoma - Cranial neuropathies ·

Leptomeningeal carcinomatosis

\begin{abstract}
Leptomeningeal carcinomatosis accounts for only $4 \%$ of cases of multiple cranial neuropathies. Here, we report the case of a patient who presented with multiple synchronous cranial neuropathies. After treatment for neuroborreliosis and broad infectious workup, endobronchial ultrasound-guided mediastinal lymph node biopsy confirmed a diagnosis of metastatic BRAF-mutated lung adenocarcinoma with leptomeningeal involvement. To our knowledge, this is the first reported case of metastatic BRAF-driven lung adenocarcinoma with leptomeningeal disease at diagnosis. In this case, the presence of leptomeningeal carcinomatosis at diagnosis, not as a late manifestation of heavily pretreated disease, alludes to a possible association between leptomeningeal involvement and BRAF-mutated non-small cell lung cancer.




\section{Case Reports in Oncology}

\begin{tabular}{l|l}
\hline Case Rep Oncol 2020;13:1258-1262 \\
\hline DOI: 10.1159/000510743 & $\begin{array}{l}\text { ○ 2020 The Author(s). Published by S. Karger AG, Basel } \\
\text { www.karger.com/cro }\end{array}$ \\
\hline
\end{tabular}

Fitzgerald et al.: Multiple Cranial Neuropathies in Metastatic BRAF-Mutated Lung Adenocarcinoma

\section{Introduction}

The differential diagnosis of multiple synchronous cranial neuropathies is broad and includes vascular, infectious, autoimmune, and malignant etiologies. In malignancy, there are several mechanisms by which multiple cranial nerves can be compromised along their course from cranial nerve nucleus to effector tissue. These include brainstem metastases involving cranial nerve nuclei, osseous compromise at cranial foramina, paraneoplastic syndromes, and compression from tumor-related hydrocephalus or leptomeningeal carcinomatosis. The latter describes malignant involvement of the pia and arachnoid mater, accounting for only $4 \%$ of cases of multiple cranial neuropathies [1]. Leptomeningeal involvement is a late, often devastating occurrence in solid tumors, most commonly breast cancer, melanoma, and nonsmall cell lung cancers (NSCLCs) [2]. Its presence at diagnosis and in untreated disease is extremely rare [2].

\section{Case Report/Case Presentation}

A 59-year-old male with extensive smoking history presented to our hospital with progressive bilateral hearing loss and right-sided peripheral facial nerve palsy (Fig. 1). Brain MRI showed contrast-enhancing parenchymal nodules as well as leptomeningeal and multiple cranial nerve enhancement (Fig. 2). Serum Lyme IgG and IgM were positive. Cerebrospinal fluid analysis from 2 separate lumbar punctures showed lymphocytic predominance with negative PCR for Lyme, normal flow cytometry, and no malignant cells by cytology. He received 4 weeks of intravenous ceftriaxone for suspected neuroborreliosis without symptom improvement during which time he experienced a 20-pound weight loss, cough, and progressive dysphagia.

Chest CT revealed bilateral hilar and mediastinal lymphadenopathy, multiple pulmonary nodules, and bilateral upper lobe cavitary lesions (Fig. 3). Infectious workup, including bronchiolar lavage with AFB staining, bacterial and fungal cultures, was unrevealing. Endobronchial ultrasound-guided biopsy of enlarged mediastinal lymph nodes revealed poorly differentiated lung adenocarcinoma. PD-L1 tumor proportion score was low/intermediate (1-49\%), and next-generation sequencing for targeted driver mutation analysis revealed the

Fig. 1. Cranial nerve exam revealing right-sided full facial nerve palsy from leptomeningeal involvement with lung adenocarcinoma.

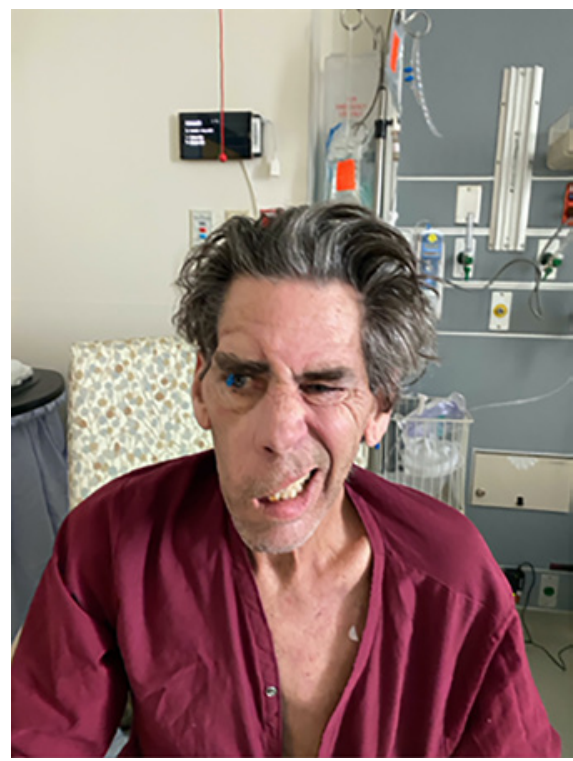




\section{Case Reports in Oncology}

Case Rep Oncol 2020;13:1258-1262 DOI: $10.1159 / 000510743$

(C) 2020 The Author(s). Published by S. Karger AG, Basel www.karger.com/cro

Fitzgerald et al.: Multiple Cranial Neuropathies in Metastatic BRAF-Mutated Lung Adenocarcinoma

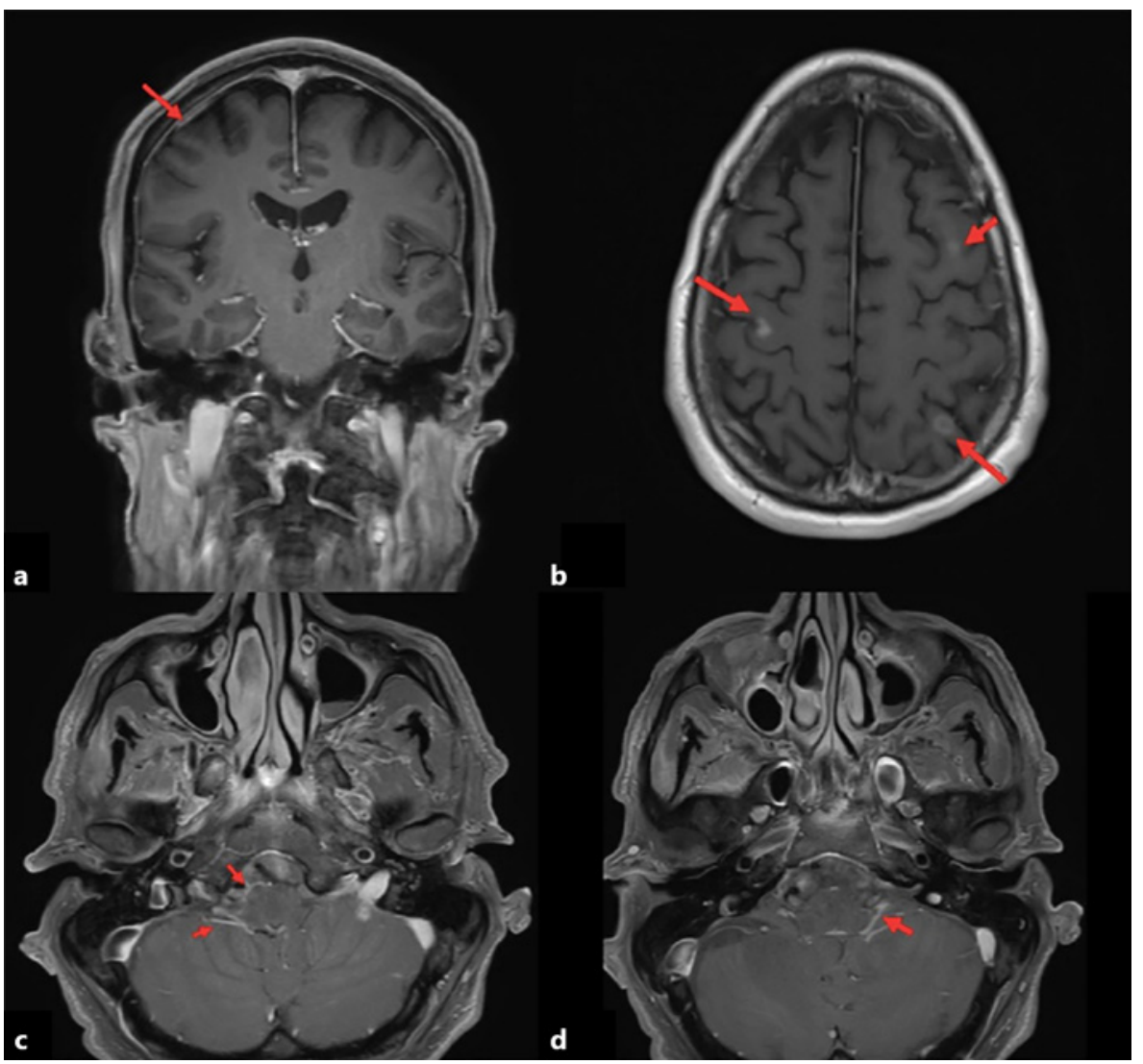

Fig. 2. Brain MRI with and without contrast demonstrating foci of leptomeningeal enhancement (a); intraparenchymal enhancement in the frontal and parietal lobes (b); bilateral cranial nerve enhancements (CN VII and VIII) suggestive of leptomeningeal carcinomatosis involving the cranial nerves (c, d).

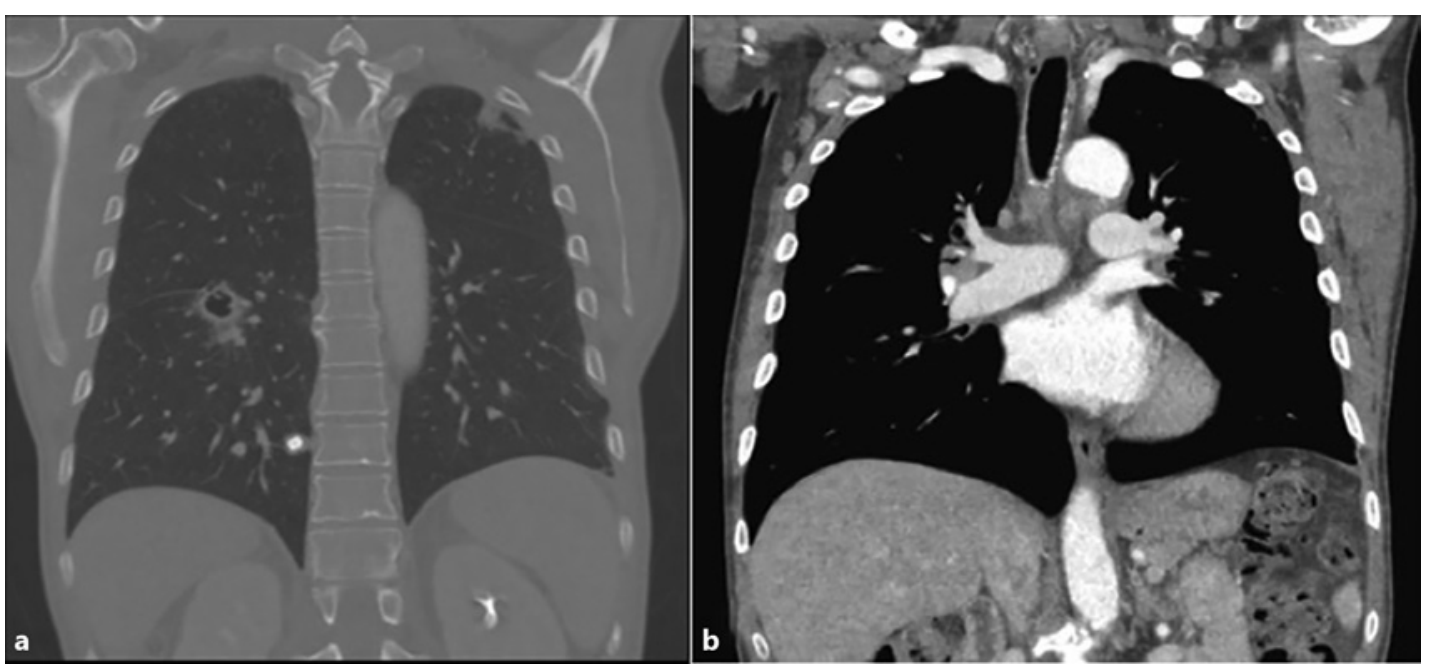

Fig. 3. CT of the chest with and without contrast demonstrating bilateral cavitary pulmonary nodules (a) and enlarged mediastinal lymph nodes (b). 
presence of BRAF V600E mutation with an allelic frequency of $36 \%$. Given rapidly progressive neurological deficits, steroids and whole-brain radiotherapy were initiated with minimal improvement. Due to pre-existing cardiomyopathy, BRAF V600E mutation-targeted therapy with dabrafenib/trametinib was deferred and he was started on ipilimumab and nivolumab. Treatment is currently on-going with clinical stability.

\section{Discussion/Conclusion}

To our knowledge, this is the first reported case of metastatic BRAF-driven lung adenocarcinoma with leptomeningeal disease at diagnosis. Moreover, it is one of few reported cases of advanced NSCLC with multiple synchronous cranial neuropathies as the presenting sign. In NSCLC, adenocarcinoma histology and lymph node metastasis predispose to malignant central nervous system involvement [3]. Some data suggest that epidermal growth factor receptor (EGFR) and anaplastic lymphoma kinase (ALK) gene aberrations are associated with a higher incidence of leptomeningeal disease [4]. However, this finding may be confounded by survivorship bias as these patient subgroups are living longer with modern targeted therapies and may be selectively at higher risk for late-stage complications. The association of leptomeningeal disease with other NSCLC driver mutations (i.e., KRAS, ROS1, BRAF, HER2, RET, and MET) has not been reported.

In this case, the presence of leptomeningeal carcinomatosis at diagnosis, not as a late manifestation of heavily pretreated disease, alludes to a possible association between leptomeningeal involvement and BRAF-mutated NSCLC. Larger observational datasets may be useful in uncovering a true relationship.

\section{Acknowledgement}

Clinical photographs included in this case are a courtesy of Dr. John Chang, VA Connecticut Healthcare System.

\section{Statement of Ethics}

The patient and his family have provided written informed consent for the use of his information and clinical images in this report, including photographs.

\section{Conflict of Interest Statement}

The authors have no conflicts of interest to declare.

\section{Funding Sources}

There is no funding source associated with this manuscript. 


\section{Case Reports in Oncology}

Case Rep Oncol 2020;13:1258-1262

\begin{tabular}{l|l}
\hline DOI: $10.1159 / 000510743$ & ( 2020 The Author(s). Published by S. Karger AG, Basel
\end{tabular} www.karger.com/cro

Fitzgerald et al.: Multiple Cranial Neuropathies in Metastatic BRAF-Mutated Lung Adenocarcinoma

\section{Author Contributions}

All authors contributed substantially to the conception of this case report. B.G.F., M.G., and G.K. took primary responsibility for drafting the manuscript with significant revision and approval by H.P. and M.R. All authors approved the final version of the manuscript.

\section{References}

1 Keane JR. Multiple cranial nerve palsies: analysis of 979 cases. Arch Neurol. 2005;62(11):1714-7.

2 Wasserstrom WR, Glass JP, Posner JB. Diagnosis and treatment of leptomeningeal metastases from solid tumors: experience with 90 patients. Cancer. 1982;49(4):759-72.

3 An N, Jing W, Wang H, Li J, Liu Y, Yu J, et al . Risk factors for brain metastases in patients with non-small-cell lung cancer. Cancer Med. 2018;7(12):6357-64.

4 Remon J, Le Rhun E, Besse B. Leptomeningeal carcinomatosis in non-small cell lung cancer patients: A continuing challenge in the personalized treatment era. Cancer Treat Rev. 2018;53:128-37. 